\title{
Effects of aquatic exercise and CES treatment on the changes of cognitive function, BDNF, IGF-1, and VEGF of persons with intellectual disabilities
}

\author{
In Ho Lee ${ }^{1}$, Eun Jung $\mathrm{Seo}^{2}$ and In Soo Lim ${ }^{1 *}$ \\ ${ }^{1}$ Department of Physical Education, Changwon National University, Gyeongnam, Korea \\ ${ }^{2}$ Daejeon University, Deajeon, Korea
}

(Received: 2014/01/20, Revised: 2014/02/12, Published online: 2014/02/14)

[Purpose] The purpose of this study was to investigate the effects of aquatic exercise and CES treatment on the cognitive function by using K-WAB and BDNF, IGF-1, and VEGF of persons with intellectual disabilities. [Methods] All subjects were 15 male with intellectual disabilities who were participating in the aquatic training program and CES treatment during 12 weeks at rehabilitation center. The subjects were divided into control group, exercise group, and exercise+CES group. Blood samples for BDNF, IGF-1, and VEGF were taken from brachial vein at rest between before and after treatment. [Results] The results are summarized as follows: Cognitive function level increased significantly in the exercise+CES group compared to those in the exercise and control group. The changes of blood IGF-1 concentration were no significant difference among groups. The changes of blood BDNF and VEGF concentration were significantly increased in exercise group and exercise+CES group than control group. However, blood BDNF and VEGF concentration were significantly difference between exercise group and exercise+CES group. [Conclusion] In conclusion, it can be concluded that CES treatment with exercise can amend cognitive function of persons with intellectual disabilities more effectively and increase of BDNF and VEGF by exercise can explain the cognitive function improvement of persons with intellectual disabilities. [Keyword] Aquatic exercise, CES, Cognitive function, BDNF, IGF-1, VEGF

\section{INTRODUCTION}

It is well established on the fact that regular exercise can help in treating various brain disorders such as depression, insomnia, Parkinson disease, and Alzheimer's disease. However in case of the patients with intellectual disabilities, they lack determination to participate in any physical exercise mainly due to decreased motor nerves activity, behavioral disorder, lack of concentration and poor communication skills. Additionally, since establishing voluntarily participations and self-motivations are difficult for the intellectual disabled patients, the effectiveness of the rehabilitation treatment is also very limited. Therefore there is a need for appropriate measure to be taken for the purpose of providing interest and motivation to the intellectually disabled patients. Considering these aspects, through unique and interesting characteristics of water, aquatic exercise could maximize the effectiveness of exercise. Since embarrassment of physical disabilities can be overcome in water, patients with intellectual disabilities can actively participate in exercising [1]. Recently, as part of effective methods of improving the cognitive functions caused by the deficiency of neurotransmitters in depression, Alzheimer's disease, stroke, and Parkinson's disease, the CES (cranial electrotherapy stimulation) equipments are given attentions to. The CES activates on the particular neurons of the electrical current in the brain stem, increasing the production of the neurotransmitters thereby increasing the electric and chemical activations [2]. The CES can be used as non-pharmaceutical intervention method for the purpose of chronic pain syndromes such as anxiety, depression, insomnia, stress relief, headache, backache, toothache, muscle pain that are reported to be largely effective in improving anxiety and pain prior to surgery [3]. Importantly, the effect of CES is related to enhanced motion performance capability in stroke patients that can be used as a tool to enhance the effectiveness of exercise during the process of rehabilitation [4-6]. Therefore

\footnotetext{
* Corresponding author: In-Soo Lim, Tel. 82-55-279-7496, Fax. 82-10-4004-5565, Email. iminsu@changwon.ac.kr

(c)2014 The Korean Society for Exercise Nutrition
} 
it is expected that the effect of the CES measure may enhances the cognitive function of the intellectually disabled.

On the other hand, the BDNF (brain-derived neuropathic factor), the IGF-1 (insulin-like growth factor), and the VEGF (vascular endothelial growth factor) that came into the recent spotlight, may increase the resistance on the death of the neurons cells and facilitates the production of cerebrovascular and nerve formations [7-9]. It is being reported that the signal control of the nerve formation in the hippocampus affecting BDNF, IGF-1 and VEGF, strengthens the neural plasticity, learning and memory and enhances the cognitive function $[10,11]$. In light of these facts, exercise of the brain with nerve disability, the CES interventions and the analysis of the BDNF, IGF-1 and VEGF could verify the rehabilitation methods and their effectiveness. Hence, in this experiment, the aquatic exercise was conducted to the mentally ill patient for the purpose of inducing interest in exercising, and the CES measures that have been known to be effective in improving cognitive function, concentration, and anxiety symptoms, were taken. Therefore the objective of this experiment is to prove the rehabilitation effect of the aquatic exercise and CES through 12 weeks of conducting aquatic exercises and by analyzing the CES measures such as cognitive function, BDNF, IGF-1, and VEGF.

\section{RESEARCH METHODS}

\section{Subjects}

The participants of this experiment are 15 intellectually disabled patients, aged between 13 17 with second to third degree of mental disability ratings which included autistic patients. They were from the 'disabled community C', students of the middle and high school swimming lessons doing as part of their special physical education program. The study composed of voluntary agreement by the caregivers of the participants. 5 patients were allocated in each group of the control group, exercise group and exercise + CES group respectively.

\section{Aquatic training program}

The aquatic exercise program was produced through expert meeting between the special physical education instructors and swimming instructors. The aquatic exercise composed of 10 minutes of preparation exercise, 30 minutes of main exercise and 10 minutes of clean-up exercise which involved total of 50 minutes, running every three times a week. Putting
Table 1. Physical characteristics of the subjects

\begin{tabular}{lccc}
\hline \multicolumn{1}{c}{ Group } & Age (years) & Height $(\mathrm{cm})$ & Weight $(\mathrm{kg})$ \\
\hline Control & $16.00 \pm 1.87$ & $153.96 \pm 12.27$ & $48.38 \pm 11.05$ \\
Exercise & $15.60 \pm 2.19$ & $153.32 \pm 8.93$ & $48.22 \pm 15.72$ \\
Exercise + CES & $15.80 \pm 3.03$ & $151.72 \pm 15.79$ & $54.00 \pm 18.93$ \\
\hline
\end{tabular}

Table 2. Aquatic training program

\begin{tabular}{llc}
\hline \multicolumn{1}{c}{ Step } & \multicolumn{1}{c}{ Aquatic exercise } & Time \\
\hline Warm-up & - stretching in water & $10 \mathrm{~min}$ \\
& - front, side, and back walking in water & \\
Main Exercise & - kicking, Knees to chest & $30 \mathrm{~min}$ \\
& - inside and outside movement of feet & \\
& - running, side step, jumping & \\
Cool-down & - free swim & $10 \mathrm{~min}$ \\
& - stretching in water & \\
\hline
\end{tabular}

focus on the actual swimming style, the aquatic program composed of leg posture, hand posture, respiration, freestyle, and backstroke. Having regards on the intensity of exercise, individual differences on the physical functions of the disabled patients were considered thereby the duration and the levels of difficulty were appropriately chosen by the instructor.

\section{CES treatment}

The CES used in the experiment was Alpha-stim 100 (Electromedical products international, USA), where the electrical current being 100 600 $\mu \mathrm{A}$, with the frequency value of $0.5,1.5,100 \mathrm{~Hz}$ (pulse/sec). The CES measures were divided into three stages of introduction, development and ending stages that composed of approximately $25 \sim 35$ minutes. In the CES manual, it is recommended to use within 600 $\mu \mathrm{A}$ range in accordance to the individual response. However considering the fact that the participants in the experiments were all intellectually disabled patients, from week $1 \sim 4$, low current of $100 \mu \mathrm{A}$ was initiated, increasing to $200 \mu \mathrm{A}$ in week $5 \sim 8$, to the maximum current of $300 \mu \mathrm{A}$ in week $9 \sim 12$.

\section{Cognitive function test}

The cognitive function test used in the experiment was the K-WAB (korean western aphasia battery), the standardized version of WAB (western aphasia battery). The cognitive function assessment provisions were the oral language test, written language test and the movement test. Oral language test comprised total of 420 points which consisted of speaking, comprehension, naming and speech repeating provisions. The written language test comprised total of 200 points which 
consisted reading and writing provisions. The movement test comprised total point of 180 , which included provisions such as action organization, space, calculation and drawing. The method of measurement was carried out by professional assessor asking questions to the participants, and then asked to follow in accordance to the instruction given which was then assessed in points through observation and recording of the answers and conducts given by the participants.

\section{Blood sampling and analysis}

For blood analysis, subjects were fasted since 10 hours prior to the experiment. $10 \mathrm{ml}$ of blood samples were taken from brachial vein at before and after training. BDNF and VEGF were analyzed by Molecular device using the ELISA method. IGF-1 was measured by the Diasorin using a LIAISON IGF-1 kit and analyzed by CLIA method.

\section{Statistics analysis}

Using the SPSS 19.0 statistical software, mean and deviance were calculated, and mean difference test was conducted through the method of two-way ANOVA with repeated measures. In case that correlation effects appear, one-way ANOVA and paired t-test were conducted. Significance level was set at .05

\section{RESULTS}

\section{Changes in cognitive function}

The changes in the cognitive function assessment data showed that there were significant differences in all groups, point in time, and cause-effect relationships $(\mathrm{p}<0.05, \mathrm{p}<$ 0.001). There was significant increase in cognitive function values in Exercise and Exercise + CES group than the controlled group $(p<0.05)$. Additionally, the exercise + CES
Table 3. Result of cognitive function among the groups (unit: point)

\begin{tabular}{lccl}
\hline \multicolumn{1}{c}{ Group } & Before & After & \multicolumn{1}{c}{ F-value } \\
\hline Control & $226.80 \pm 32.29$ & $234.00 \pm 27.01$ & Group : 5.33* \\
Exercise & $217.20 \pm 40.77$ & $248.00 \pm 28.53$ & Time : 17.00*** \\
Exercise + CES & $260.00 \pm 45.26$ & $303.00 \pm 29.65$ & G $\times$ T : $3.57^{*}$ \\
\hline
\end{tabular}

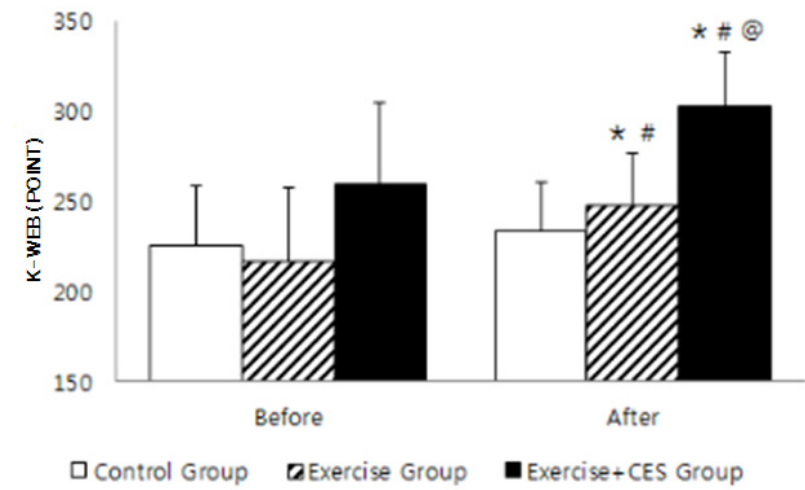

Fig. 1. The changes of cognitive function among the groups. $* p<.05$ significantly different from before treatment, ${ }^{\#} \mathrm{p}<.05$ significantly different from control group in after treatment, ${ }^{a} \mathrm{p}<.05$ significantly different from exercise group in after treatment.

group showed significant increase in the value when compared to the exercise along group $(\mathrm{p}<0.05)$.

\section{Changes in neurotransmitters}

The results of the changes in the neurotransmitters between groups are listed in the Table 4 below.

The changes in the BDNF assessment data showed that there were significant differences in all groups, point in time, and cause-effect relationships $(\mathrm{p}<0.05, \mathrm{p}<0.001)$. Although there were no significant differences within the groups before and after trainings when compared with the controlled group, there was a significant difference between the Exercise group and the Exercise + CES group $(p<0.05)$. Even the comparison data between each groups, despite the fact that the Exercise and Exercise + CES intervention groups showed

Table 4. Result of neurotransmitters among the groups

\begin{tabular}{|c|c|c|c|c|}
\hline Factor & Group & Before & After & F-value \\
\hline \multirow[t]{3}{*}{$\mathrm{BDNF}(\mathrm{pg} / \mathrm{mL})$} & Control & $20220.6 \pm 249$ & $20939.0 \pm 37$ & \multirow{3}{*}{$\begin{array}{l}\text { Group : } 5.96^{*} \\
\text { Time : } 115.17^{* * *} \\
\mathrm{G} \times \mathrm{T}: 23.31^{* *}\end{array}$} \\
\hline & Exercise & $19756.8 \pm 108$ & $30155.0 \pm 371$ & \\
\hline & Exercise + CES & $19894.4 \pm 320$ & $30304.4 \pm 315$ & \\
\hline \multirow[t]{3}{*}{ IGF-1 (ng/mL) } & Control & $251.80 \pm 30.49$ & $253.20 \pm 10.80$ & \multirow{3}{*}{$\begin{array}{l}\text { Group : } 3.542 \\
\text { Time : } 2.71 \\
\mathrm{G} \times \mathrm{T}: 0.50\end{array}$} \\
\hline & Exercise & $241.00 \pm 42.82$ & $255.40 \pm 41.18$ & \\
\hline & Exercise + CES & $203.20 \pm 20.63$ & $216.00 \pm 21.90$ & \\
\hline \multirow[t]{3}{*}{ VEGF (pg/mL) } & Control & $215.60 \pm 43.43$ & $224.20 \pm 43.16$ & \multirow{3}{*}{$\begin{array}{l}\text { Group : } 5.72^{* *} \\
\text { Time : } 221.79^{* * *} \\
\mathrm{G} \times \mathrm{T}: 46.14^{* * *}\end{array}$} \\
\hline & Exercise & $211.00 \pm 27.01$ & $344.80 \pm 22.43$ & \\
\hline & Exercise + CES & $202.00 \pm 31.47$ & $346.40 \pm 21.83$ & \\
\hline
\end{tabular}




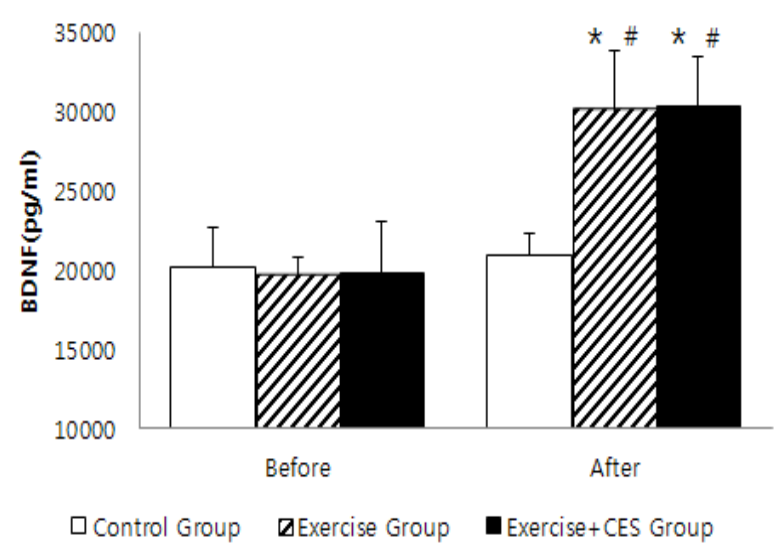

Fig. 2. The changes of BDNF among the groups. $* p<.05$ significantly different from before treatment, ${ }^{\#} \mathrm{p}<.05$ significantly different from control group in after treatment

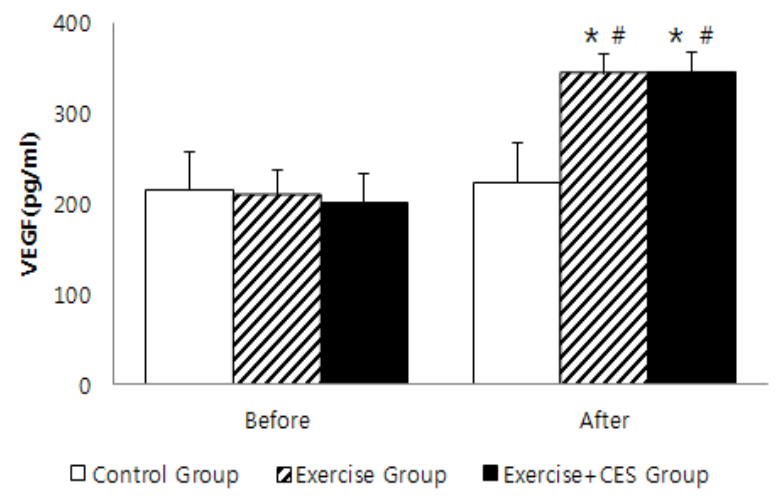

Fig. 3. The changes of VEGF among the groups. $* p<0.05$ significantly different from before treatment, ${ }^{\#} \mathrm{p}<0.05$ significantly different from control group in after treatment

significant increase than the controlled group $(\mathrm{p}<0.05)$, there was no significant difference observed when compared between the Exercise and Exercise + CES groups $(p<0.05)$.

The changes in the IGF-1 assessment data showed that there were no significant differences in all groups, point in time, and cause-effect relationships $(p>0.05)$. The changes in the VEGF assessment data showed that there were significant differences in all groups, point in time, and cause-effect relationships $(\mathrm{p}<0.05, \mathrm{p}<0.001)$. In another word, there were significant differences in before and after treatments between the Exercise and Exercise + CES groups $(p<0.05)$. Even the comparison data between each group, despite the fact that the Exercise and Exercise + CES intervention groups showed significant increase than the controlled group, there was no significant difference observed when compared between the Exercise and Exercise + CES groups

\section{DISCUSSION}

In this experiment, the effect of aquatic exercise and CES measures on the changes of cognitive functions, BDNF, IGF-1, VEGF, and serotonin values were analyzed and discussed accordingly. According to the Wilson et al. [12], in relation to exercise and cognitive function, it has been reported that exercise stimulates the biochemical changes of the nerves in the brain which induce the activation of the neurotransmitters and formation of the genetics thereby affecting the proliferation of the neurons and their survival resulting in the enhancement of learning, memory and cognitive function skills. However through which mechanism that exercising affects the brain and its cognitive function remains unknown. In this experiment, the cognitive functions of the Exercise group and Exercise + CES group increased after the treatment group than before treatment. According to the Wilson et al. [12], the reduction in the cognitive function was highly correlated to the reduction of the physical functions. Salmons $\&$ Sahakian [13] showed that low concentration and attention deficiency characteristics that are common in patients with brain disabilities improved through regular exercise. Especially in the Foster et al. [14], similar result was suggested where cognitive function was enhanced through regular exercise. It has been shown that the cognitive function was greatly increased in the Exercise + CES group than the Exercise group alone hence additional CES intervention was identified to be helpful in enhancing the cognitive function of the patients with intellectual disabilities. It is therefore estimated that the CES interventions stimulates the tiny current in the brain neurons which boost the electric and chemical activations thereby enhancing the cognitive function of the intellectually disabled.

The changes in the BDNF in the experiment showed significant increase by $52.6 \%$ in the Exercise group, and 52.3\% in the Exercise + CES group. However the CES measures were found to be ineffective as there was no significant difference identified between the Exercise group and Exercise + CES group. According to the Seifert et al. [15], it has been suggested that the BDNF concentration was increased in the brain after 3 month of exercising, and the Ferris et al. [16] and Hillman et al. [17] studies have reported that the brain function was increased including cognitive function resulted by the BDNF increase through exercising. Analyzing the correlation between the cognitive function and the BDNF from this experiment, static correlation $(\mathrm{r}=0.45, \mathrm{p}<0.05)$ was identified where increased BDNF was found to enhance the cognitive function skills. It is thereby suggested that regular exercise increase the level of the BDNF, which 
enhances the cognitive function of the brain. Additionally, in the experiment, the changes in the IGF-1 level in Exercise group and Exercise + CES group increased after treatment in comparison with the before treatment, yet the difference was insignificant. Eliakin [18] have suggested that there was a sudden increase in the IGF-1 level immediately after exercise, and Berg \& Bang [19] study have reported that the IGF-1 concentration started to increase at 5 minutes of exercising where the peak concentration was reached at approximately 20 minutes. Considering these aspects, it is suggested that the changes in the IGF-1 level in one-off exercise reacts more vigorously in accordance with the intensity of exercise, than the level that has been stabilized before and after the treatment. On the other hand, the experiment showed significant increase in the VEGF by $63.4 \%$ in the Exercise group, and $71.4 \%$ in the Exercise + CES group. However, additional effect of CES measures could not be confirmed as there was no significant difference between the Exercise and Exercise + CES group. According to the Franzoni et al. [20], regular exercise was found to be effective in increasing the function of the endotheliocytes by increasing the productivity of the VEGF. The Bloor [21], and Prior et al. [22] studies have identified that the increased level of VEFG enhances the blood flow rate of the internal aorta and arteriole which would helps the blood supply and vascularization through peripheral reduction in the blood vessel resistance which is associated with the brain cognitive function. From the experiment, the aquatic exercise increased the VEGF level in the brain that positively affected the brain vascular cells and blood flow rate, which could explain why there had been an increased cognitive function. As follows, the aquatic exercise has been shown to be effective in improving cognitive function of the intellectually disabled patients and additional CES interventions was shown to improve even in greater effect in the cognitive function skills. Additionally, regular exercise increased the concentration of BDNF and VEGF where as BDNF and VEGF level increased, the cognitive function skill too increased.

\section{CONCLUSION}

The purpose of this study was to investigate the effects of aquatic exercise and CES treatment on the cognitive function by using K-WAB and BDNF, IGF-1, and VEGF of persons with intellectual disabilities. Cognitive function level were significantly increased in exercise + CES group than exercise group and control group. The changes of blood IGF-1 concentration were no significant difference among groups. The changes of blood BDNF and VEGF concentration were signi- ficantly increased in exercise group and exercise + CES group than control group. As a result, it can be explain that exercise helps cognitive function of persons with intellectual disabilities and micro-current stimulation with CES treatment further improves cognitive function by activating nerve cell. In addition, it can be suggested that the increment of BDNF by exercise stimulates the production of brain cells and the increase of VEGF by exercise accelerates the production of endothelial cells. In conclusion, it can be concluded that CES treatment with exercise can amend cognitive function of persons with intellectual disabilities more effectively and increase of BDNF and VEGF by exercise can explain the cognitive function improvement of persons with intellectual disabilities.

\section{REFERENCES}

[1] Lim IS, Chan JW. The effects of aquatic training on physical function, information processing ability, and blood lipid in stroke patients, Korean Journal of Physical Education. 2003:42(4):647-654.

[2] Taylor DN, Lee CT, Katims JJ. Effect of cranial transcutaneous electrical nerve simulation in normal subjects at rest and during psychoiogical stress. Acupunct Electrother Res. 1991;16:65-74.

[3] Kirsch DL, Smith RB. The use of cranial electrotherapy stimulation in the management of chronic pain : a review. Neuro-Rehabilitation. 2000;14(2):85-94

[4] Fregni F, Boggio, PS, Mansur CG, Wagner T, Ferreira MJ, Lima MC. Transcranial direct current stimulation of the unaffected hemisphere in stroke patients. Neuroreport. 2005;16(14):1551-1555.

[5] Kincses TZ, Antal A, Nitsche MA, Bartfai O, Paulus W. Facilitation of probabilistic classification learning by transcranial direct current stimulation of the prefrontal cortex in the human. Neuropsychologia. 2004;42(1): 113-117.

[6] Kirsch DL CES for mild traumatic brain injury. Pranctical Pain Management. 2006;60-64.

[7] Komulainen P, Pedersen M, Hanninen T, Bruunsgaard H, Lakka TA, Kivipelto M. BDNF is a novel marker of cognitive function in aging women: the DR's extra Study. Neurobiol Learn Mem. 2008;90(4):596-603.

[8] Landi F, Capoluongo E, Russo A, Onder G, Vessari M, Lulli P, Minucci A, Pahor AM, Suppi C, Bemabei R. Free insulin-like growth factor-1 and cognitive function in older persons living in community. Growth hormone \& IGF-1 Resesrch. 2007;17(1):58-66. 
[9] Zoladz JA, Pilc A, Majerczak J, Grandys M, ZapartBukowska J, Duda K. Endurance training increases plasma brain-derived neurotrophic factor concentration in young healthy men. J Physiol Pharmacol. 2008;59(7): 119-132.

[10] Burchett SA, Hicks TP. The mysterious trace amines: protean neuromodulators of synaptic transmission in mammalian brain. Progress in neurobiology. 2006;79(5): 223-246.

[11] Karlsson RM, Holmes A. Galanin as a modulater of anxiety and depression and a therapeutic target for affective disease. Amino Acids. 2006;31(3):231-239

[12] Wilson R, Schneider J, Bienias J, Evans D, Bennett D. Parkinsonian like signs and risk of incident Alzheimer disease in older persons. Archives of Neurology. 2003;60(4): 539-544.

[13] Salmond CH, Sahakian BJ. Cognitive outcome in traumatic brain injury survivors. Current Opinion in Critical Care. 2005;11(2):111-116.

[14] Foster PO, Rosenblatt KP, Kulji RO. Exercise-induced cognitive plasticity, implications for mild cognitive impairment and Alzheimer's disease. Front. Neurology. 2011;2(28):1-15.

[15] Seifert T, Brassard P, Wissenberg M, Rasmussen $P$, Nordby P, Stalknecht B, Adser H, Jakobsen AH, Pilegaard $\mathrm{H}$, Nielsen $\mathrm{HB}$, Secher $\mathrm{NH}$. Endurance training enhances
BDNF release from the human brain. Am J Physiol. Regul Integr Comp Physiol. 2010;298(2):327-377.

[16] Ferris LT, Williams JS, Shen, CL. The effect of acute exercise on serum brain-derived neurotrophic factor levels and cognitive function. Medicine \& Science in Sports \& Exercise. 2007;39(4):728-734.

[17] Hillman CH, Erickson KI, Kramer AF. Be smart, exercise your heart: exercise effect on brain and cognition. Nature Reviews Neuroscience 2008;9: 58-65.

[18] Eliakin A, Brasel JA, Cooper DM. GH response to exercise: Assesment of the pituitary period, and relationship with circulation components of the GH-IGF-1 Axis in adolescent females. J Pediart Endocrinol Metab. 1999;12(1): 47-56.

[19] Berg U, Bang P. Exercise and circulating insulin-like growth factor I. Hormone Research in Paediatrics. 2005; 62:50-58.

[20] Franzoni F, Ghiadoni L, Galetta F, Plantinga Y, Lubrano V, Huang Y. Physical activity, plasma antioxidant capacity, and endothelium-dependent vasodilation in young and older men. Am J Hyper. 2005;18:510-516.

[21] Bloor CM. Angiogenesis during exercise and training. Angiogenesis. 2005;8(3):263-271.

[22] Prior BM, Yang HT, Terjung RL. What makes vessels grow with exercise training? J Appl Physiol. 2004;97(3): 1119-1128. 